\title{
Fuhrman Grade 2
}

National Cancer Institute

\section{Source}

National Cancer Institute. Fuhrman Grade 2. NCI Thesaurus. Code C62429.

Nuclei slightly irregular, approximately 15 microns; nucleoli evident. 